\title{
Convergent Generalized Monotone Splitting of Matrices*
}

\author{
By O. L. Mangasarian
}

\begin{abstract}
Let $B$ and $T$ be $n \times n$ real matrices and $r$ an $n$-vector and consider the system $u=B T u+r$. A new sufficient condition is given for the existence of a solution and convergence of a monotone process to a solution. The monotone process is a generalization of the Collatz-Schröder procedure.
\end{abstract}

1. Introduction. Collatz-Schröder [1] consider the system

$$
u=T u+r,
$$

where $T$ is a given $n \times n$ real matrix and $r$ a given $n$-vector, and prescribe the monotone iterative process

$$
\left[\begin{array}{c}
v^{i+1} \\
w^{i+1}
\end{array}\right]=\left[\begin{array}{rr}
T_{1} & -T_{2} \\
-T_{2} & T_{1}
\end{array}\right]\left[\begin{array}{l}
v^{i} \\
w^{i}
\end{array}\right]+\left[\begin{array}{l}
r \\
r
\end{array}\right], \quad i=0,1,2, \cdots,
$$

where $T=T_{1}-T_{2}, T_{1} \geqq 0$ and $T_{2} \geqq 0$. A sufficient condition for the monotonicity and convergence of the above process is the existence of initial $0^{0}, w^{0}$ satisfying

$$
v^{0} \leqq v^{1}, \quad w^{1} \leqq w^{0}, \quad v^{0} \leqq w^{0},
$$

where $v^{1}, w^{1}$ are computed from (1.2). Condition (1.3) guarantees that (1.1) has a solution $u$ such that

$$
v^{0} \leqq v^{1} \leqq \cdots \leqq v^{i} \leqq \cdots \leqq u \leqq \cdots \leqq w^{i} \leqq \cdots \leqq w^{1} \leqq w^{0}
$$

and $u=\lim _{i \rightarrow \infty}\left(v^{i}+w^{i}\right) / 2$.

In this work, we consider the system

$$
u=B T u+r
$$

where $B$ is some $n \times n$ real nonsingular matrix, and prescribe the iterative process (2.3). Here, however, the splitting $T=T_{1}-T_{2}$ is not monotonic with respect to the nonnegative orthant but with respect to the dual cone generated by the rows of $B^{-1}$, that is:

$$
B^{-1} y \geqq 0 \Rightarrow T_{1} y \geqq 0 \text { and } T_{2} y \geqq 0 .
$$

In Collatz-Schröder [1], $B^{-1}=I$. A sufficient condition for the monotonicity and convergence of the iteration (2.3) is the existence of $v^{0}, w^{0}$ satisfying (2.5). Condition (2.5) guarantees that (1.5) has a solution satisfying $B^{-1} v^{i} \leqq B^{-1} u \leqq B^{-1} w^{i}$ and $u=\lim _{i \rightarrow \infty}\left(v^{i}+w^{i}\right) / 2$.

Received March 18, 1971.

AMS 1970 subject classifications. Primary 65F10; Secondary 15A39.

Key words and phrases. Monotone splitting of matrices, linear inequalities.

* Supported by the National Science Foundation Grant GJ-362 and the United States Army Contract No. DA-31-124-ARO-D-462. 
By using Motzkin's theorem of the alternative for linear inequalities [4], [3], a sufficient condition (3.2) for (2.5) can be obtained. This condition insures the existence of a solution $u$ to (1.5) and the convergence and monotonicity of the iterative process (2.3). When applied to $A u=b$, condition (3.2) gives existence results such as (3.6): If $A \geqq-I$, and $A^{\prime} x \geqq 0, x \geqq 0$ implies that $b x=0$, then $A u=b$ has a solution. (Here and throughout, a prime denotes the transpose.)

A reason for considering the system (1.5) instead of (1.1) is that many of the classical iterative schemes for solving the system $A u=b$, where $A$ is a given $n \times n$ real matrix and $b$ is a given $n \times 1$ real vector, consist of splitting the matrix $A$ into the difference of two $n \times n$ real matrices, $A=M-N$, and using the equivalent system $u=M^{-1} N u+M^{-1} b$ to derive the iteration

$$
u^{i+1}=M^{-1} N u^{i}+M^{-1} b .
$$

The Jacobi, Gauss-Seidel and successive overrelaxation methods [5, Chapter 3] fall into this category. If we make the identifications $B=M^{-1} N, r=M^{-1} b$, we obtain (1.5), the system under consideration in this work. The present results, then, give a sufficient condition for the monotonicity and convergence of the process (2.3) which can be associated with any of the above classical schemes.

2. The Monotone Splitting and its Convergence. We consider here the problem

$$
u=B T u+r
$$

where $B$ and $T$ are given $n \times n$ real matrices with $B$ nonsingular, and $r$ is a given $n$-vector. We split the matrix $T$ as follows:

$$
T=T_{1}-T_{2},
$$

and require that

$$
B^{-1} y \geqq 0 \Rightarrow T_{1} y \geqq 0 \quad \text { and } \quad T_{2} y \geqq 0,
$$

or, equivalently [2], we require that

$$
T_{1} B \geqq 0 \text { and } T_{2} B \geqq 0 .
$$

(To see the equivalence of (2.2a) and (2.2b), we note that if (2.2b) holds, then, $B^{-1} y \geqq 0$ implies $T_{1} B B^{-1} y=T_{1} y \geqq 0$. Conversely, if (2.2a) holds, then, $B^{-1} B=I \geqq 0$ implies $T_{1} B \geqq 0$.)

We consider the iteration

$$
\left[\begin{array}{c}
v^{i+1} \\
w^{i+1}
\end{array}\right]=\left[\begin{array}{ll}
B & 0 \\
0 & B
\end{array}\right]\left[\begin{array}{rr}
T_{1} & -T_{2} \\
-T_{2} & T_{1}
\end{array}\right]\left[\begin{array}{l}
v^{i} \\
w^{i}
\end{array}\right]+\left[\begin{array}{l}
r \\
r
\end{array}\right]
$$

and begin by establishing the following result.

(2.4) Convergence AND Existence TheOrem. Let (2.1) and (2.2) hold. If there exist $v^{0}, w^{0}$ in $R^{n}$ such that

$$
\left[\begin{array}{cc}
B^{-1} & 0 \\
0 & B^{-1}
\end{array}\right]\left[\begin{array}{c}
v^{1}-v^{0} \\
w^{0}-w^{1}
\end{array}\right] \geqq 0, \quad B^{-1}\left(w^{0}-v^{0}\right) \geqq 0,
$$

with $v^{1}, w^{1}$ computed from (2.3), then 


$$
\left[\begin{array}{cc}
B^{-1} & 0 \\
0 & B^{-1}
\end{array}\right]\left[\begin{array}{c}
v^{i+1}-v^{i} \\
w^{i}-w^{i+1}
\end{array}\right] \geqq 0, \quad B^{-1}\left(w^{i}-v^{i}\right) \geqq 0
$$

for $i=0,1,2, \cdots$. In addition, the system $u=B T u+r$ has a solution $u$ such that $B^{-1} v^{i} \leqq B^{-1} u \leqq B^{-1} w^{i}$ and $u=\lim _{i \rightarrow \infty}\left(v^{i}+w^{i}\right) / 2$.

Remark. If we set $B=I$, then we obtain the results of Collatz-Schröder [1].

Proof. We first establish (2.6) by induction. Because of (2.5), (2.6) holds for $i=0$. Assume now that (2.6) holds for $i$ and proceed to show that it also holds for $i+1$.

$$
\begin{aligned}
{\left[\begin{array}{cc}
B^{-1} & 0 \\
0 & -B^{-1}
\end{array}\right]\left[\begin{array}{c}
v^{i+2}-v^{i+1} \\
w^{i+2}-w^{i+1}
\end{array}\right] } \\
=\left[\begin{array}{ll}
T_{1} & T_{2} \\
T_{2} & T_{1}
\end{array}\right]\left[\begin{array}{c}
v^{i+1} \\
-w^{i+1}
\end{array}\right]+\left[\begin{array}{cc}
B^{-1} & 0 \\
0 & B^{-1}
\end{array}\right]\left[\begin{array}{r}
r \\
-r
\end{array}\right]-\left[\begin{array}{cc}
B^{-1} & 0 \\
0 & B^{-1}
\end{array}\right]\left[\begin{array}{c}
v^{i+1} \\
-w^{i+1}
\end{array}\right] \\
\geqq\left[\begin{array}{ll}
T_{1} & T_{2} \\
T_{2} & T_{1}
\end{array}\right]\left[\begin{array}{c}
v^{i} \\
-w^{i}
\end{array}\right]+\left[\begin{array}{cc}
B^{-1} & 0 \\
0 & B^{-1}
\end{array}\right]\left[\begin{array}{c}
r \\
-r
\end{array}\right]-\left[\begin{array}{cc}
B^{-1} & 0 \\
0 & B^{-1}
\end{array}\right]\left[\begin{array}{c}
v^{i+1} \\
-w^{i+1}
\end{array}\right]
\end{aligned}
$$$$
=0
$$

We also have

$$
\begin{aligned}
B^{-1}\left(w^{i+1}-v^{i+1}\right) & =\left(T_{1}+T_{2}\right)\left(w^{i}-v^{i}\right) & & \text { (by (2.3)) } \\
& \geqq 0 & & \text { (by (2.6) and (2.2a)). }
\end{aligned}
$$

Hence, (2.6) holds for $i+1$ and the induction is complete. We now have from (2.6) that

$$
B^{-1} v^{0} \leqq B^{-1} v^{1} \leqq \cdots \leqq B^{-1} v^{i} \leqq \cdots \leqq B^{-1} w^{i} \leqq \cdots \leqq B^{-1} w^{1} \leqq B^{-1} w^{0} .
$$

Hence, the monotone sequences $\left\{B_{k}^{-1} v^{i}\right\}$ and $\left\{B_{k}^{-1} w^{i}\right\}$ have limits $a_{k}^{*}$ and $b_{k}^{*}$, so that also the vector sequences $\left\{B^{-1} v^{i}\right\}$ and $\left\{B^{-1} w^{i}\right\}$ have limits $a^{*}$ and $b^{*}$. From the continuity of the linear operator $B$, we have also that the vector sequences $\left\{0^{i}\right\}$ and $\left\{w^{i}\right\}$ converge to $0=B a^{*}$ and $w=B b^{*}$. Hence, from (2.3),

$$
\begin{aligned}
B^{-1} v & =T_{1} v-T_{2} w+B^{-1} r, \\
B^{-1} w & =-T_{2} v+T_{1} w+B^{-1} r .
\end{aligned}
$$

By letting $u=(v+w) / 2$, we have that

$$
B^{-1} u=\left(T_{1}-T_{2}\right) u+B^{-1} r=T u+B^{-1} r .
$$

That $B^{-1} v^{i} \leqq B^{-1} u \leqq B^{-1} w^{i}$ follows from

$$
B^{-1} u=B^{-1}\left(\frac{v+w}{2}\right)=\frac{a^{*}+b^{*}}{2} .
$$

3. Sufficient Conditions for Solving $u=B T u+r$. By using Motzkin's theorem of the alternative [4], [3], we now give a sufficient condition for the existence of $v^{0}, w^{0}$ satisfying (2.5) and hence for the existence of a solution of $u=B T u+r$ and the convergence of the iterative process (2.3). 
(3.1) CONVERGENCE AND EXISTENCE THEOREM. Theorem (2.4) holds with assumption (2.5) replaced by

$$
\left.\begin{array}{rl}
\left(-I+T_{1} B\right)^{\prime} x+\left(T_{2} B\right)^{\prime} y & \geqq 0, \\
\left(T_{2} B\right)^{\prime} x+\left(-I+T_{1} B\right)^{\prime} y & \geqq 0, \\
x, y & \geqq 0,
\end{array}\right\} \Rightarrow x B^{-1} r=0 .
$$

Proof. We have to show that (3.2) implies (2.5). Now (3.2) implies that

$$
\left.\begin{array}{rl}
\left(-I+T_{1} B\right)^{\prime} x+\left(T_{2} B\right)^{\prime} y & \geqq 0, \\
\left(T_{2} B\right)^{\prime} x+\left(-I+T_{1} B\right)^{\prime} y & \geqq 0, \\
-x B^{-1} r+y B^{-1} r & >0, \\
x, y & \geqq 0,
\end{array}\right\} \quad \text { has no solution }(x, y)
$$

which implies that

$$
\left.\begin{array}{r}
\left(-B^{-1}+T_{1}\right)^{\prime} x+T_{2}^{\prime} y-\left(B^{-1}\right)^{\prime} z=0, \\
-T_{2}^{\prime} x-\left(-B^{-1}+T_{1}\right)^{\prime} y+\left(B^{-1}\right)^{\prime} z=0, \\
\left(B^{-1} r\right)^{\prime} x-\left(B^{-1} r\right)^{\prime} y+\eta=0, \\
x, y, z \geqq 0 \\
\eta>0
\end{array}\right\} \quad \text { has no solution }(x, y, z, \eta)
$$

which by Motzkin's theorem is equivalent to

$$
\left.\begin{array}{rl}
\left(-B^{-1}+T_{1}\right) v^{0}-T_{2} w^{0}+B^{-1} r \zeta & \geqq 0, \\
T_{2} v^{0}-\left(-B^{-1}+T_{1}\right) w^{0}-B^{-1} r \zeta & \geqq 0, \\
-B^{-1} v^{0}+B^{-1} w^{0} & \geqq 0, \\
\zeta & >0
\end{array}\right\} \quad \text { has a solution }\left(v^{0}, w^{0}, \zeta\right)
$$

which is equivalent to

$$
\left.\begin{array}{r}
T_{1} v^{0}-T_{2} w^{0}+B^{-1} r-B^{-1} v^{0} \geqq 0, \\
B^{-1} w^{0}+T_{2} v^{0}-T_{1} w^{0}-B^{-1} r \geqq 0, \\
B^{-1}\left(w^{0}-v^{0}\right) \geqq 0,
\end{array}\right\} \quad \text { has a solution }\left(v^{0}, w^{0}\right)
$$

which is equivalent to (2.5) having a solution. Q.E.D.

By observing that if the system $u=B T u+r$ has a solution $\bar{u}$ then the system $-u=-B T u+r$ has a solution $-\bar{u}$, we obtain the following result from Theorem (3.1) by appropriate modifications.

(3.3) CONVERGENCE AND EXISTENCE TheOREM. Let (2.1), (2.2) and (3.2) hold. Then, there exist $v^{0}, w^{0}$ such that

$$
\left[\begin{array}{cc}
B^{-1} & 0 \\
0 & B^{-1}
\end{array}\right]\left[\begin{array}{c}
v^{1}-v^{0} \\
w^{0}-w^{1}
\end{array}\right] \leqq 0, \quad B^{-1}\left(w^{0}-v^{0}\right) \leqq 0,
$$

where $v^{1}, w^{1}$ are computed from the iteration 


$$
\left[\begin{array}{c}
v^{i+1} \\
w^{i+1}
\end{array}\right]=\left[\begin{array}{ll}
B & 0 \\
0 & B
\end{array}\right]\left[\begin{array}{rr}
T_{1} & -T_{2} \\
-T_{2} & T_{1}
\end{array}\right]\left[\begin{array}{c}
v^{i} \\
w^{i}
\end{array}\right]-\left[\begin{array}{l}
r \\
r
\end{array}\right], \quad i=0,1,2, \cdots,
$$

which produces $v^{i}, w^{i}$ satisfying

$$
\left[\begin{array}{cc}
B^{-1} & 0 \\
0 & B^{-1}
\end{array}\right]\left[\begin{array}{c}
v^{i+1}-v^{i} \\
w^{i}-w^{i+1}
\end{array}\right] \leqq 0, \quad B^{-1}\left(w^{i}-v^{i}\right) \leqq 0 .
$$

In addition, the system $-u=-B T u+r$ has a solution $u$ such that $B^{-1} v^{i} \geqq B^{-1} u \geqq$ $B^{-1} w^{i}$ and $u=\lim _{i \rightarrow \infty}\left(v^{i}+w^{i}\right) / 2$.

The following convergence and existence result for $A u=b$ is obtained from Theorem (3.3) above by setting $T_{2}=0, A=-B^{-1}+T_{1}$ and $b=B^{-1} r$.

(3.4) Convergence AND Existence fOR $A u=b$. Consider the system $A u=b$ where $A$ is a given $n \times n$ matrix and $b$ is a given vector. Assume that $A B+I \geqq 0$ for some nonsingular $n \times n$ matrix $B$. If

$$
\left.\begin{array}{rl}
(A B)^{\prime} x & \geqq 0, \\
x & \geqq 0,
\end{array}\right\} \quad \Rightarrow b x=0,
$$

then $A u=b$ has a solution $u$. This solution can be obtained from the iteration

$$
\left[\begin{array}{l}
v^{i+1} \\
w^{i+1}
\end{array}\right]=\left[\begin{array}{l}
B\left(T_{1} v^{i}-b\right) \\
B\left(T_{1} w^{i}-b\right)
\end{array}\right], \quad i=0,1,2, \cdots,
$$

where $T_{1}=A+B^{-1}$, starting with $v^{0}, w^{0}$ which exist and satisfy

$$
B^{-1}\left(v^{1}-v^{0}\right) \leqq 0, \quad B^{-1}\left(w^{0}-w^{1}\right) \leqq 0, \quad B^{-1}\left(w^{0}-v^{0}\right) \leqq 0 .
$$

This iterative process produces $v^{i}, w^{i}$ satisfying

$$
\begin{gathered}
B^{-1}\left(v^{i+1}-v^{i}\right) \leqq 0, \quad B^{-1}\left(w^{i}-w^{i+1}\right) \leqq 0, \quad B^{-1}\left(w^{i}-v^{i}\right) \leqq 0, \\
B^{-1} v^{i} \geqq B^{-1} u \geqq B^{-1} w^{i} \quad \text { and } \quad u=\lim _{i \rightarrow \infty}\left(v^{i}+w^{i}\right) / 2 .
\end{gathered}
$$

(3.5) Corollary. If we take $B=I$ in (3.4), we have that for $A \geqq-I$, if $A^{\prime} x \geqq 0$, $x \geqq 0$ implies $b x=0$, then $A u=b$ has $a$ solution, and the iteration and monotonicity relations of (3.4) simplify accordingly. Similarly, if we take $B=-I$, we have that for $A \leqq I$, if $A^{\prime} x \leqq 0, x \geqq 0$ implies $b x=0$, then $A u=b$ has $a$ solution.

Computer Sciences Department

The University of Wisconsin

Madison, Wisconsin 53706

1. L. Collatz, Funktionalanalysis und numerische Mathematik, Die Grundlehren der math. Wissenschaften, Band 120, Springer-Verlag, Berlin, 1964; English transl., Academic Press, New York, 1966, pp. 350-390. MR 34 \#4961.

2. O. L. MANGASARIAN, "Characterizations of real matrices of monotone kind," SIAM Rev., v. 10, 1968, pp. 439-441. MR 38 \#5818.

3. O. L. Mangasarian, Nonlinear Programming, McGraw-Hill, New York, 1969, pp. 28-29. MR 40 \#5263.

4. T. S. MotZKIN, Beiträge zur Theorie der Linearen Ungleichungen, Inaugural Dissertation, Basel, Jerusalem, 1936.

5. R. S. VARgA, Matrix Iterative Analysis, Prentice-Hall, Englewood Cliffs, N. J., 1962. MR 28 \# 1725. 